\title{
Complementing structural information of modular proteins with small angle neutron scattering and contrast variation
}

\author{
J. G. Grossmann · A. J. Callaghan • M. J. Marcaida • \\ B. F. Luisi · F. H. Alcock · K. Tokatlidis • M. Moulin • \\ M. Haertlein $\cdot$ P. Timmins
}

Published online: 26 March 2008

(c) EBSA 2008

\section{Erratum to: Eur Biophys J DOI 10.1007/s00249-008-0278-z}

Three additional authors have to be added to the list of authors. The names and affilliations are as follows:

M. Moulin · M. Haertlein · P. Timmins Institut Laue Langevin, 6, Rue Jules Horowitz, BP 156, 38042 Grenoble Cedex 09, France

The online version of the original article can be found under doi:10.1007/s00249-008-0278-z.

J. G. Grossmann $(\bowtie)$

Molecular Biophysics Group, STFC Daresbury Laboratory,

Daresbury Science and Innovation Campus,

Warrington, Cheshire WA4 AD, UK

e-mail: j.g.grossmann@dl.ac.uk

A. J. Callaghan · M. J. Marcaida - B. F. Luisi

Department of Biochemistry, University of Cambridge,

80 Tennis Court Road, Cambridge CB2 1GA, UK

F. H. Alcock $\cdot$ K. Tokatlidis

Institute of Molecular Biology and Biotechnology,

Foundation for Research and Technology Hellas,

PO Box 1385, Heraklion, 71110 Crete, Greece

M. Moulin · M. Haertlein · P. Timmins

Institut Laue Langevin, 6, Rue Jules Horowitz,

BP 156, 38042 Grenoble Cedex 09, France

Present Address:

A. J. Callaghan

Biophysics Laboratories, Institute of Biomedical

and Biomolecular Science, University of Portsmouth,

King Henry Building, King Henry 1st Street,

Portsmouth PO1 2DY, UK
In additon, correct acknowledgments are as follows:

\section{Acknowledgments}

The work on RNase E was supported by the Wellcome Trust. We thank Martyn Symmons and Martin Moncrieffe for stimulating discussions and invaluable advice. The work on TIM10 was supported by funds from IMBBFORTH. The ILL and STFC Daresbury Laboratory are acknowledged for beamtime. This work benefited from the activities of the DLAB (Deuteration Laboratory) consortium funded by the European Union under contract HPRI2001-50065 and from United Kingdom Engineering and Physical Sciences Research Council (EPSRC)-funded activity within the ILL-EMBL Deuteration Laboratory (DLAB) under Grant GR/R99393/01. 